UVX 2012, 01013 (2013)

DOI: $10.1051 /$ uvx/201301013

(C) Owned by the authors, published by EDP Sciences, 2013

\title{
Génération d'un faisceau polarisé gamma à très haut flux par diffusion Compton : l'expérience Mighty Laser
}

\author{
I. Chaikovska ${ }^{1}$, R. Chiche ${ }^{1}$, E. Cormier ${ }^{2}$, N. Delerue ${ }^{1}$, D. Jehanno ${ }^{1}$, \\ F. Labaye ${ }^{1,2}$, M. Lacroix ${ }^{1}$, V. Soskov ${ }^{1}$, A. Variola ${ }^{1}$ et F. Zomer ${ }^{1}$ \\ 1 Univ. Paris-sud 11, Laboratoire de l'Accélérateur Linéaire, CNRS, IN2P3, UMR 8607, \\ 91898 Orsay Cedex, France \\ 2 Univ. Bordeaux1, Centre Lasers Intenses et Applications, CNRS, CEA, UMR 5107, \\ 33405 Talence, France
}

\begin{abstract}
Résumé. Dans le cadre de la recherche et du développement de sources de rayons gamma polarisés, une cavité Fabry-Perot (FP) non planaire et un système laser de forte puissance moyenne ont été développés et utilisés sur un accélérateur de particules afin de produire un rayonnement gamma par diffusion Compton inverse. La conception du système laser et de la cavité FP est présentée ainsi que les méthodes de synchronisation nécessaires.
\end{abstract}

\section{INTRODUCTION}

Dans la gamme d'énergie allant $\mathrm{du} \mathrm{KeV}$ à quelques dizaines de $\mathrm{MeV}$, des développements de sources de photons de flux allant de $10^{10}$ à $10^{14}$ photons de largeur spectrale $\frac{\Delta \omega}{\omega} \sim 10 \%$ sont actuellement en cours pour de nombreuses applications. L'imagerie médicale [1], la radiothérapie [2], la coronarographie [3] ainsi que l'étude d'œuvres d'art [4] nécessitent des rayons x d'une énergie allant d'une dizaine de keV à $100 \mathrm{keV}$ alors que la gestion des déchets nucléaires [5] et leur surveillance requièrent des rayons $\gamma$ de quelques MeV. D'autre part, les applications en physique des hautes énergies [6] nécessitent des rayons $\gamma$ de $20 \mathrm{MeV}$ à quelques centaines de $\mathrm{GeV}$ et tout particulièrement dans le contexte des projets de collisionneurs ILC [7] et CLIC [8] où un faisceau $\gamma$ polarisé circulairement pourrait être utilisé pour créer des positrons polarisés longitudinalement $[6,9,10]$. L'expérience Mighty Laser se situe dans le cadre de ces collisionneurs.

Différentes méthodes sont à ce jour utilisées : le rayonnement synchrotron couplé à des ondulateurs et les tubes à anodes tournantes. Cependant, elles ont plusieurs inconvénients comme la taille et les coûts pour les synchrotrons et le flux limité pour les tubes. La diffusion Compton inverse lors de la collision d'un photon et d'un électron constitue une méthode alternative pour produire ces photons de haute énergie. C'est le seul processus physique permettant de faire passer l'énergie d'un photon de $1 \mathrm{eV}$ (laser infrarouge) à plusieurs keV voir GeV. D'autre part, ce processus peut présenter une monochromaticité importante. Enfin, sous certaines conditions, l'émission peut être polarisée. Cependant, cette approche souffre d'une très faible section efficace de diffusion. Elle nécessite donc de très grands flux d'électrons et/ou de photons.

Une première approche de mise en œuvre de la diffusion Compton à haut flux consiste à utiliser des lasers de très forte énergie qui ont l'inconvénient du coût, de la taille et de la basse cadence. Nous proposons une alternative mettant en œuvre l'amplification passive d'un laser de forte puissance moyenne dans une cavité FP [11]. Cette approche vise à empiler de manière cohérente les impulsions

This is an Open Access article distributed under the terms of the Creative Commons Attribution License 2.0, which permits unrestricted use, distribution, and reproduction in any medium, provided the original work is properly cited. 
Web of Conferences

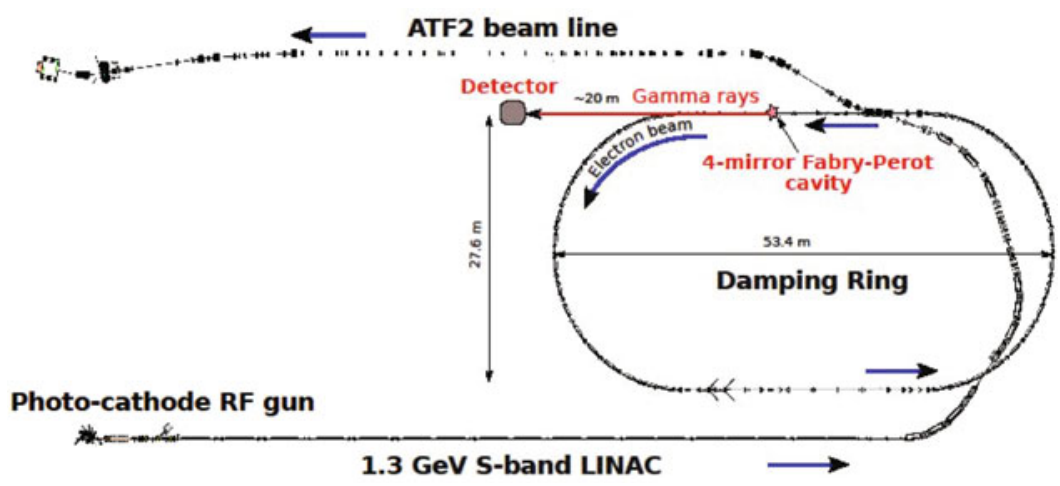

Figure 1. Agencement de l'Accelerator Test Facility à KEK. L'étoile rouge montre la position du système optique décrit dans la «Section 2 » et la flèche rouge indique la direction de propagation des rayons $\gamma$ produits qui passeront au travers de plusieurs collimateurs afin de sélectionner ceux de plus hautes énergies.

du laser au sein de la cavité FP, le champ électrique de chaque impulsion incidente venant s'additionner à celui oscillant dans le résonateur à la condition que leur phase soit identique. Il a été démontré qu'elle permet d'obtenir des gains supérieurs à 100000 en mode continu et 10000 en mode pulsé ce qui permettrait d'atteindre des puissances moyennes intra-cavité de plusieurs centaines de kilowatts pour une puissance incidente de quelques dizaines de watts. C'est dans ce cadre qu'est développé le projet Mighty Laser qui est présenté dans cet article et dont l'objectif final est la génération d'un faisceau de $\gamma$ polarisés circulairement à très haut flux sur l'anneau de stockage de l'Accelerator Test Facility (ATF) à KEK au Japon [12] comme montré sur la « Figure $1 »$.

\section{LE SYSTÈME OPTIQUE}

\subsection{L'amplification laser}

Dans le but d'obtenir la circulation d'un très grand nombre de photons laser au sein d'une cavité FP, le faisceau laser incident dont les impulsions seront empilées dans la cavité FP doit lui aussi présenter une puissance moyenne importante. Deux approches sont possibles. La première consiste à utiliser un oscillateur de forte puissance moyenne dont les impulsions seront directement injectées dans la cavité FP alors que la seconde consiste en l'utilisation d'un oscillateur laser basse puissance moyenne dont le faisceau laser sera préalablement amplifié dans un système d'amplification d'impulsions à dérive de fréquence fibré (FCPA) avant l'injection dans la cavité FP. Cependant, pour délivrer un faisceau adéquat à l'empilement cohérent d'impulsions dans la cavité FP, l'oscillateur doit fournir un peigne de fréquence avec une fréquence de répétition $f_{\text {rép }}$ et une phase entre la porteuse et l'enveloppe $\Delta \varphi_{C E}$ extrêmement stables [13]. La puissance moyenne requise par le projet dépassant largement celle des oscillateurs bas bruit commerciaux, la seconde approche est choisie.

La « Figure $2 »$ présente le système optique utilisé durant l'expérience. Celui-ci consiste en un oscillateur commercial modifié (Origami-10 de Onefive $\mathrm{GmbH}$ ) délivrant des impulsions laser de 200 fs limitées par transformée de Fourier et cadencées à 178,5 MHz. Afin de limiter les non-linéarités induites lors du processus d'amplification, les impulsions laser sont étirées dans un réseau de Bragg en volume (CVBG de OptiGrate) [14]. Ce CVBG agit aussi comme un filtre spectral, faisant passer la largeur spectrale de $5 \mathrm{~nm}$ à $2,2 \mathrm{~nm}$. Le faisceau passe ensuite dans un modulateur électro-optique afin de créer la modulation nécessaire à l'asservissement de l'oscillateur sur la cavité FP [15] qui sera détaillé dans la « Section 3 ». Ces impulsions laser sont ensuite amplifiées dans une fibre optique dopée à large cœur (NKT Photonics DC-200/40) optiquement pompée par une diode laser multi-mode. La puissance 


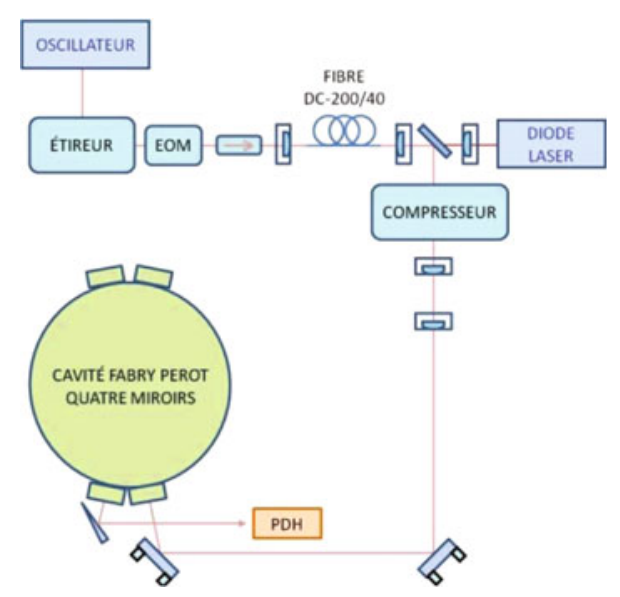

Figure 2. Synoptique du système optique.

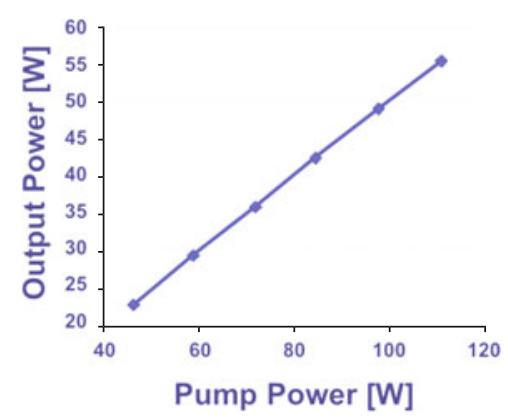

Figure 3. Puissance moyenne extraite de la fibre optique dopée en fonction de la puissance moyenne du pompage optique.

moyenne extraite varie linéairement avec la puissance de pompe et l'efficacité est de $51 \%$ comme le montre la « Figure 3 ». Après amplification, les impulsions sont comprimées à environ 68 ps dans un second CVBG de dispersion différente du premier. L'intensité du faisceau laser dans ce CVBG est assez faible pour ne pas entrainer de non-linéarités. Un télescope permet finalement d'adapter les dimensions du faisceau à celles du mode théorique circulant dans la cavité.

\subsection{La cavité Fabry-Perot}

La diffusion Compton inverse illustrée sur la « Figure 4 » est la diffusion inélastique d'un électron sur un photon, lui transmettant ainsi une partie de son énergie. Cette interaction est décrite précisément dans [16] et seuls les éléments nécessaires seront exposés.

Le flux de $\gamma$ émis durant le processus de diffusion Compton dépend de la section efficace de l'interaction ainsi que de la luminosité des collisions [17]. En supposant des distributions Gaussiennes ainsi qu'une taille constante pour les paquets d'électrons et le faisceau laser, celle-ci est donnée par la formule suivante :

$$
\mathcal{L}=N_{\theta} N_{\rho k} f_{\text {rép }} \frac{\cos (\phi / 2)}{\sqrt{\sigma_{y \theta}^{2}}+\sigma_{y \rho k}^{2}} \frac{1}{\sqrt{\left(\sigma_{x \theta}^{2}+\sigma_{x \rho k}^{2}\right) \cos ^{2}(\phi / 2)+\left(\sigma_{z \theta}^{2}+\sigma_{z \rho k}^{2}\right) \sin ^{2}(\phi / 2)}}
$$




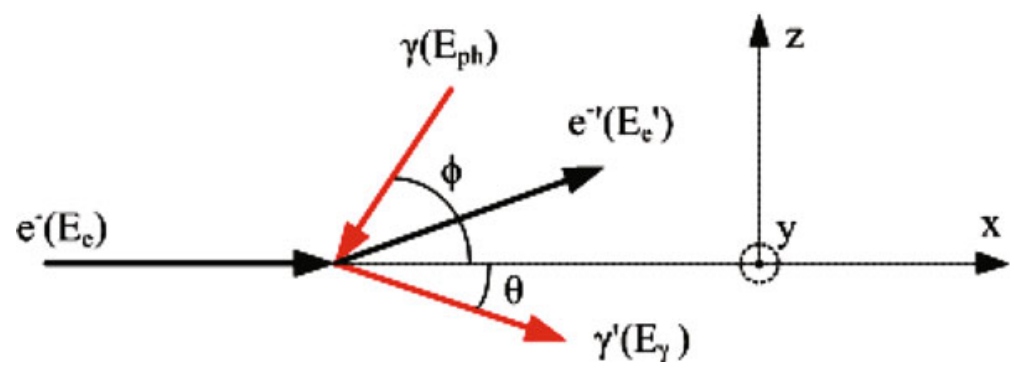

Figure 4. Diffusion Compton inverse d'un électron d'énergie $E_{e}$ sur un photon $\gamma$ d'énergie $E_{p h}$ avec un angle d'interaction $\phi$. Le photon $\gamma$ ' diffusé avec un angle $\theta$ possède une énergie $E_{\gamma}$.

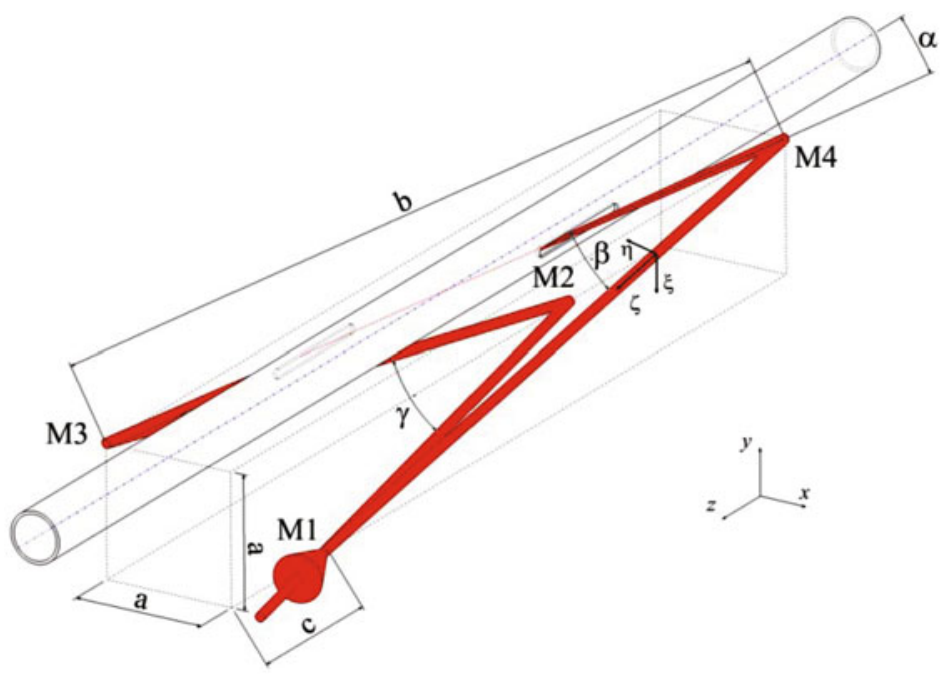

Figure 5. Schéma de la cavité quatre miroirs installée sur l’ATF.

où $N_{\theta}$ et $N_{\rho k}$ sont les nombres d'électrons dans les paquets et de photons dans les impulsions laser, respectivement et $\sigma_{x \theta}^{2}$ et $\sigma_{x \rho k}^{2}$ indiquent les tailles des paquets d'électrons et du faisceau laser, respectivement. Afin d'optimiser le flux de $\gamma$, il est nécessaire de réduire la taille du faisceau laser ainsi que l'angle de collision. Le design de la cavité FP doit prendre en compte ces paramètres.

Les cavités FP constituées de deux miroirs concaves ne sont pas stables pour une utilisation avec de petites tailles de mode [11]. Le choix s'est donc porté sur une cavité à quatre miroirs. De plus, dans le cadre de la création positrons polarisés longitudinalement, les rayons $\gamma$ doivent être polarisés circulairement. Par conséquent, afin d'avoir des modes propres polarisés circulairement, la géométrie tétraédrique de la « Figure 5 » est choisie [18].

M1, M2, M3 et M4 représentent respectivement les deux miroirs plans et les deux miroirs sphériques de rayon de courbure $500 \mathrm{~mm}$. Un parallélépipède est défini par $a=70 \mathrm{~mm}$ et $b=500 \mathrm{~mm}$. Les miroirs M3 et M4 se situent à proximité de ses coins et les miroirs M1 et M2 en sont décalés de $c=81,19 \mathrm{~mm}$. Des fentes de $5 \mathrm{~mm}$ de large sont découpées dans le tube où circule le faisceau d'électrons afin de propager le faisceau laser vers le point d'impact. L'angle d'intersection entre les deux faisceaux est $\alpha=8,05^{\circ}$. Pour finir, $\beta / 2=6,25^{\circ}$ et $\gamma / 2=7,6^{\circ}$ sont les deux angles d'incidences sur M1, M2 et M3, M4, respectivement. 


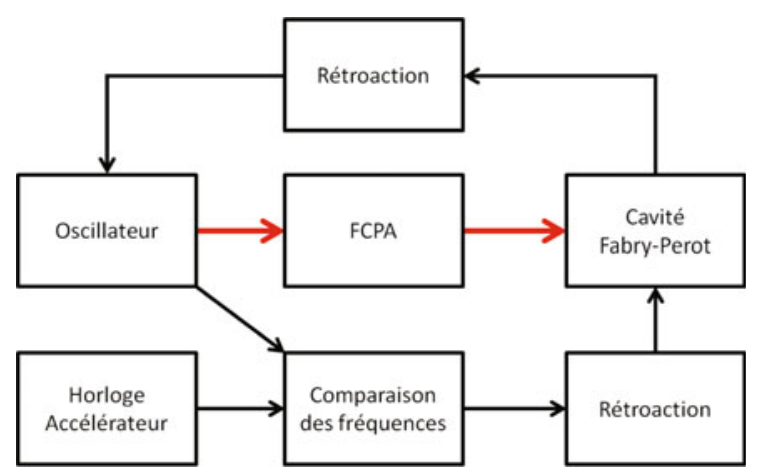

Figure 6. Synoptique du système d'asservissement.

\section{SYNCHRONISATION}

Il a été indiqué dans la «Section 2 » qu'une grande stabilité était requise au niveau de l'oscillateur laser afin que le processus d'empilement d'impulsions ait lieu au sein de la cavité FP. Or, malgré tout le soin apporté aux conceptions de la cavité FP et de l'oscillateur laser, le niveau de stabilité requis sur l'accord entre les cavités laser et Fabry-Perot pour obtenir un fort gain dans cette dernière est trop important pour pouvoir se passer d'un système de stabilisation actif de la cavité laser. Pour cela, nous avons choisit la méthode Pound-Drever-Hall (PDH) [15] pour la production d'un signal d'erreur et un système d'asservissement à base d'intégrateurs agissant sur un actuateur dans l'oscillateur laser.

Des simulations de la variation du couplage dans la cavité en fonction de $\Delta \varphi_{C E}$ pour une fréquence de répétition $f_{\text {rép }}$ asservie dans une cavité FP de finesse 3000 ont montré une perte négligeable ce qui nous dispense d'asservir $\Delta \varphi_{C E}$.

En plus de la synchronisation entre l'oscillateur laser et la cavité FP, il est important que le système présenté ici soit synchronisé sur l'horloge de l'ATF afin que la fréquence de répétition du faisceau laser au sein de la cavité FP reste un entier de fois celle de l'anneau de stockage de l'ATF. Le système présente donc un second système de synchronisation. Un diagramme simplifié du système d'asservissement est présenté « Figure 6 »

Pour finir, la table optique est posé sur trois pieds motorisés permettant de la déplacer horizontalement et verticalement avec une précision de $1 \mu \mathrm{m}$ afin de superposer le faisceau laser et le faisceau d'électrons pour avoir un recouvrement spatial optimal.

\section{RÉSULTATS}

Le système a été installé en août 2010 sur l'ATF et les premiers rayons $\gamma$ ont été produits en octobre de la même année. Plusieurs sessions de prise de données ont été effectuées puis analysées. Celles-ci sont expliquées en détails dans [19] et seulement les points principaux seront explicités ici. Après les collisions, les rayons $\gamma$ se propagent colinéairement au faisceau d'électrons jusqu'à ce que ceux-ci soient déviés par les électro-aimants. Ils passent ensuite dans différent collimateurs avant d'interagir avec le détecteur et des rayons $\gamma$ ont pu être obtenus. La génération des rayons $\gamma$ a été simulée avec le code de collisions CAIN2.40 en y insérant les paramètres mesurés des deux faisceaux interagissant (voir les tableaux 1). Les spectres d'énergie mesurés et simulés des rayons $\gamma$ obtenus sont représentés sur la Figure 7 et l'on peut voir une très bonne corrélation entre les deux spectres.

Comme il a été indiqué dans l'introduction, pour beaucoup d'applications nécessitant des rayons $\mathrm{x}$ ou $\gamma$, une des caractéristiques importante est le flux de photons produits. Le « Tableaux 2 » résume les plus importants flux obtenus pour différents modes de remplissage de l'anneau de stockage de l'ATF. 


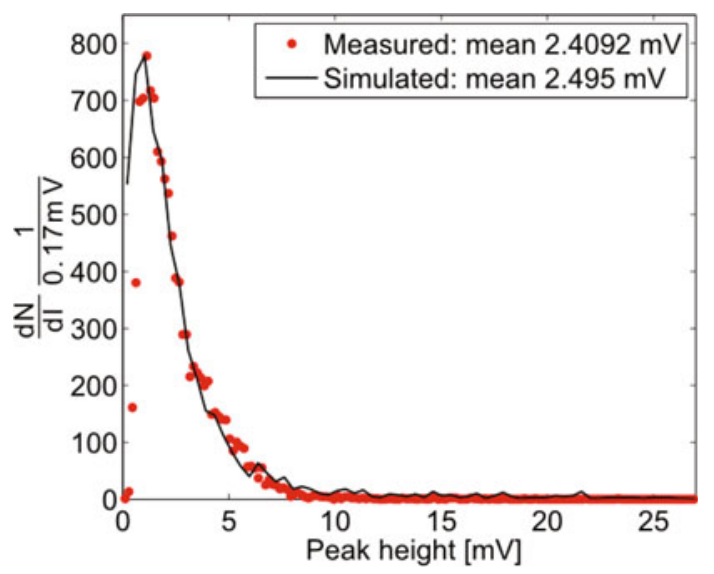

Figure 7. Comparaison entre les spectres d'énergie mesurés et simulés des rayons $\gamma$ produits. Les disques rouges représentent le spectre mesuré et la ligne noire représente le spectre simulé pour un taux de $2,7 \gamma$ produits par collision.

Tableau 1. Paramètres de l'anneau de stockage de l'ATF et du système laser durant les prises de données.

\begin{tabular}{|c|c|c|c|}
\hline \multicolumn{2}{|c|}{ Paramètres de l'anneau de stockage de l'ATF } & \multicolumn{2}{|l|}{ Paramètres du système laser } \\
\hline $\begin{array}{l}\text { Energie des électrons, } \\
E_{e}\end{array}$ & $1,28 \mathrm{GeV}$ & $\begin{array}{l}\text { Energie des } \\
\text { photons, } E_{p h}\end{array}$ & $1,2 \mathrm{eV}(1032 \mathrm{~nm})$ \\
\hline $\begin{array}{l}\text { Nombre d'électrons } \\
\text { par paquets, } N_{e}\end{array}$ & $\approx 0.5 \times 10^{10}$ & $\begin{array}{l}\text { Taille du faisceau } \\
\text { au point d'interaction }\end{array}$ & $26 / 38 \mu \mathrm{m}$ \\
\hline $\begin{array}{l}\text { Longueur des paquets } \\
\text { d'électrons, } \sigma_{z} / c=\tau_{e}\end{array}$ & $\approx 20 \mathrm{ps}$ & $\begin{array}{l}\text { Fréquence } \\
\text { de répétition, } f_{\text {rép }}\end{array}$ & $178,5 \mathrm{MHz}$ \\
\hline $\begin{array}{l}\text { Dimensions du faisceau } \\
\text { faisceau d'électrons, } \sigma_{x} / \sigma_{y}\end{array}$ & $\approx 110 / 10 \mu \mathrm{m}$ & $\begin{array}{l}\text { Finesse de la } \\
\text { cavité FP, } F\end{array}$ & $\approx 3000$ \\
\hline $\begin{array}{l}\text { Période de } \\
\text { révolution, } T_{0}\end{array}$ & $462 \mathrm{~ns}$ & $\begin{array}{l}\text { Durée des } \\
\text { impulsions }\end{array}$ & $68 \mathrm{ps}$ \\
\hline $\begin{array}{l}\text { Emittance, } \\
\gamma \varepsilon_{x / y}\end{array}$ & $\begin{array}{l}5 \times 10^{-6} / 3 \times 10^{-8} \\
\text { m.rad }\end{array}$ & $\begin{array}{l}\text { Puissance moyenne stockée } \\
\text { dans la cavité Fabry-Perot, } P_{l}\end{array}$ & $\approx 160 \mathrm{~W}$ \\
\hline
\end{tabular}

Tableau 2. Flux de rayons $\gamma$ obtenus les plus hauts.

\begin{tabular}{|l|c|c|c|c|c|}
\hline $\begin{array}{l}\text { Structure des } \\
\text { paquets d'e }\end{array}$ & $\begin{array}{c}\text { Intensité totale } \\
\text { sur } \mathbf{0 . 2} \mathbf{~ m s}\end{array}$ & $\begin{array}{c}\text { Energie déposée } \\
\text { sur } \mathbf{0 . 2} \mathbf{~ m s}\end{array}$ & $\begin{array}{c}\text { Flux intégré } \\
\text { sur } \mathbf{0 . 2} \mathbf{~ m s}\end{array}$ & $\begin{array}{c}\text { Flux intégré } \\
\text { sur 1 s }\end{array}$ & $\begin{array}{c}\text { Erreur } \\
\text { systématique }\end{array}$ \\
\hline 1 train & $893 \mathrm{mV}$ & $23750 \mathrm{MeV}$ & $990 \gamma$ & $\approx 4,9 \times 10^{6} \gamma$ & $7 \%$ \\
\hline 2 trains & $910 \mathrm{mV}$ & $24210 \mathrm{MeV}$ & $1010 \gamma$ & $\approx 5,0 \times 10^{6} \gamma$ & $7 \%$ \\
\hline 3 trains & $1010 \mathrm{mV}$ & $26800 \mathrm{MeV}$ & $1120 \gamma$ & $\approx 5,6 \times 10^{6} \gamma$ & $7 \%$ \\
\hline
\end{tabular}

\section{CONCLUSIONS ET PERSPECTIVES}

Le projet Migthy Laser a démontré la production de $\gamma$ en utilisant une cavité Fabry-Perot quatre miroirs non-planaire ainsi qu' un système d'amplificateur laser à fibre dopée. Une moyenne de 2,7 $\gamma$ sont produits à chaque collision ce qui est en accord avec les simulations effectuées sous CAIN. L'expérience a été brutalement stoppée par le tremblement de terre qui a touché le Japon le 11 mars 2011 et qui a largement endommagé l'accélérateur. Depuis, une nouvelle architecture d'amplification a été développée. Un 


\section{UVX 2012}

asservissement à une cavité de finesse 30000 en mode pulsé a aussi été réalisé. Ces deux améliorations seront installées sur l'ATF à nouveau opérationnel durant l'automne 2012.

\section{Références}

[1] F.E. Caroll et al., Am. J. Roentgenol. 179, 583-590 (2002)

[2] M.-C. Biston et al., Cancer Res. 64, 2317-2323 (2004)

[3] P. Suorti et al., Phys. Med. Biol. 48 R1-R35 (2003)

[4] M. Cotte et al., Anal.Chem. 79 6988-6994 (2007)

[5] R. Hajima et al., J. Nucl. Sci. Technol. 45 441-451 (2008)

[6] G. Moortgat-Pick et al., Phys. Rep. 460 131-243 (2008)

[7] J. Brau et al., ILC Reference Design Report Volume 1 - Executive Summary, arXiv:0712.1950

[8] CLIC PHYSICS WORKING GROUP collaboration, E. Accomando et al., Physics at the CLIC multi-TeV linear collider, hep-ph/0412251

[9] Source positrons S. Araki et al., Conceptual design of a polarised positron source based on laser Compton scattering, physics/0509016

[10] O. Dadoun et al. The Baseline Positron Production and Capture Scheme for CLIC, 1st International Particle Accelerator Conference: IPAC'10, Kyoto Japan, May 23-28 2010

[11] H. Kogelnick et al., Appl. Opt. 5, 1550-1567 (1966)

[12] F. Hinode et al., Atf accelerator test facility design and study report no. 4, KEK, Tsukuba, Japan 1995, http://1cdev.kek.jp/ATF/Pub/KEK-I-95-4.pdf

[13] T. Udem et al. Nature 416 233-237 (2002)

[14] K-H. Liao et al., Large-aperture chirped volume bragg grating based fiber cpa system, Opt. Expr. 15 (2007) 4876

[15] R.W.P. Drever et al., Appl. Phys. B 31 97-105 (1983)

[16] P. Sprangle et al., J. Appl. Phys. 72, 5032-5034 (1992)

[17] T. Suzuki, General formulae of luminosity for various types of colliding beam machines, KEK note 76-3, KEK, Tsukuba Japan (1976)

[18] F. Zomer et al., Appl. Opt. 486651 (2009)

[19] T. Akagi et al. J. INST 701021 (2012) 